Supporting Information

\title{
Development of advanced chimeric endolysin to control multidrug- resistant Staphylococcus aureus through domain shuffling
}

Chanyoung Lee a,b,1, Jinwoo Kim $^{\mathrm{a}, \mathrm{b}, 1}$, Bokyung Son ${ }^{\mathrm{a}, 2}$, and Sangryeol Ryu $\mathbf{u}^{\mathrm{a}, \mathrm{b}, \mathrm{c}}$ *

aDepartment of Food and Animal Biotechnology, Research Institute of Agriculture and Life Sciences, Seoul National University, Seoul 08826, Republic of Korea

${ }^{b}$ Department of Agricultural Biotechnology, College of Agriculture and Life Sciences, Seoul National University, Seoul 08826, Republic of Korea

${ }^{\mathrm{c} C e n t e r}$ for Food and Bioconvergence, Seoul National University, Seoul 08826, Republic of Korea

*Corresponding author.

E-mail address: sangryu@snu.ac.kr (Dr. Sangryeol Ryu)

${ }^{1}$ The first two authors contributed equally to the work.

${ }^{2}$ Current address: Gene Expression and Regulation Section, Laboratory of Cell and Molecular Biology, National Institute of Diabetes and Digestive and Kidney Diseases, National Institutes of Health, Bethesda, MD, USA 


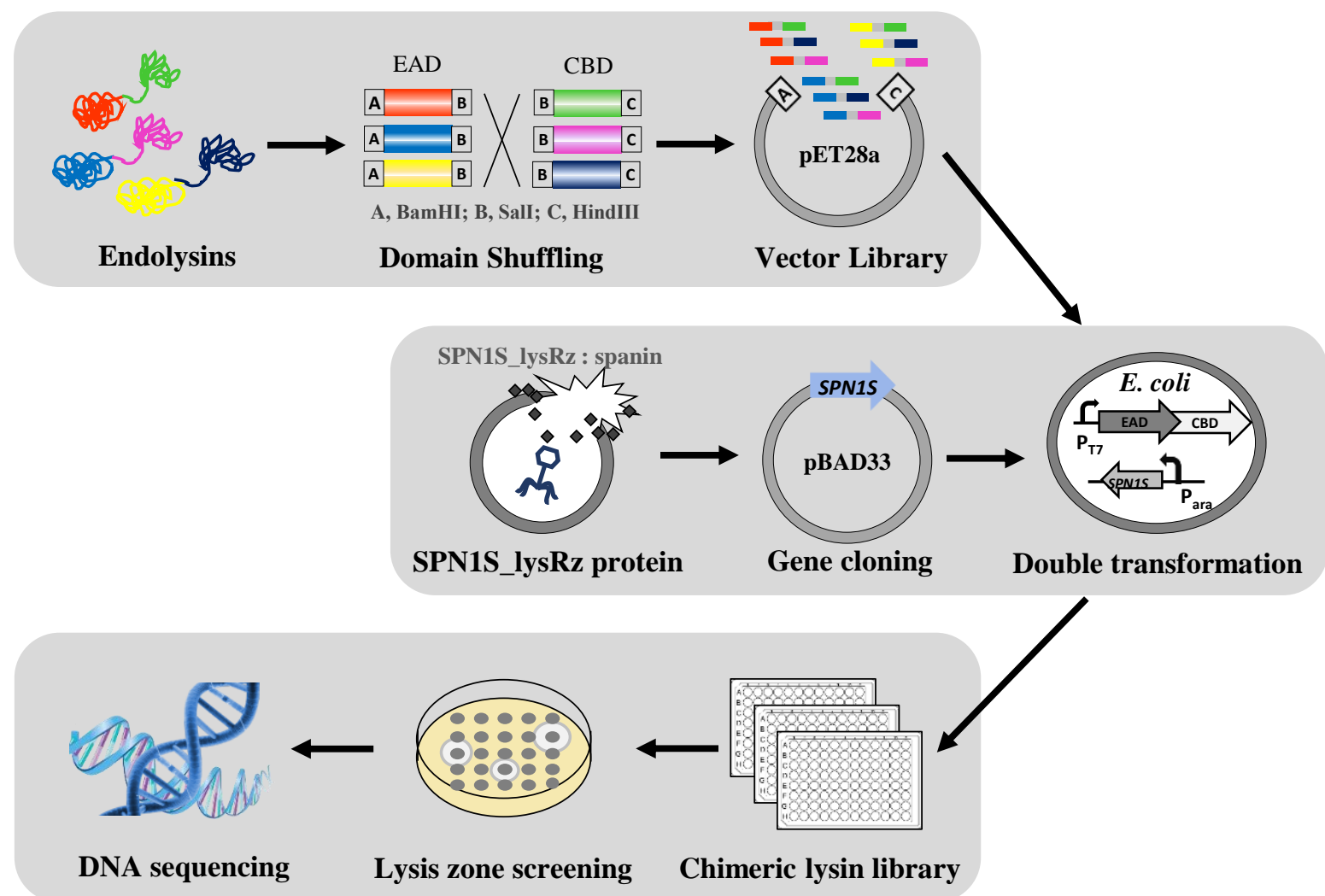

Figure S1. Overview of a chimeric endolysin library construction and rapid screening method. 


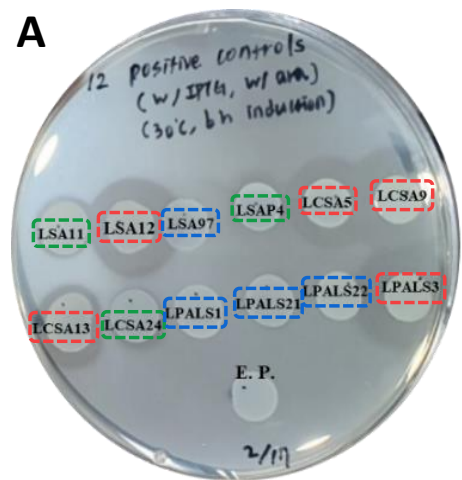

Group L (Large lysis zone)

Group M (Medium lysis zone)

Group S (Small lysis zone)
B

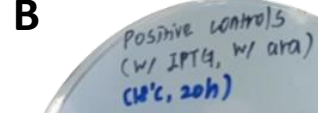
$\left(n^{\prime} c, 20 h\right)$
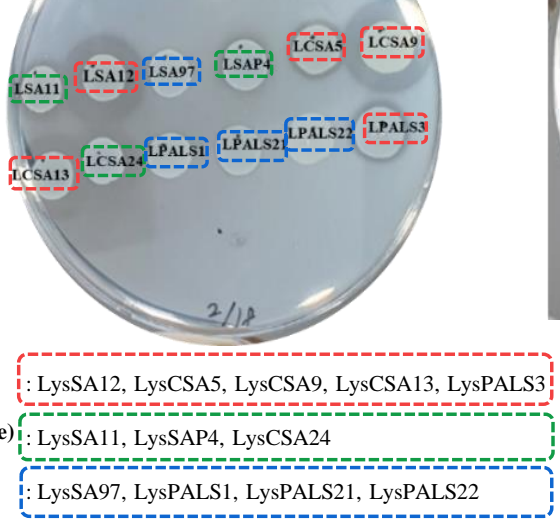

C

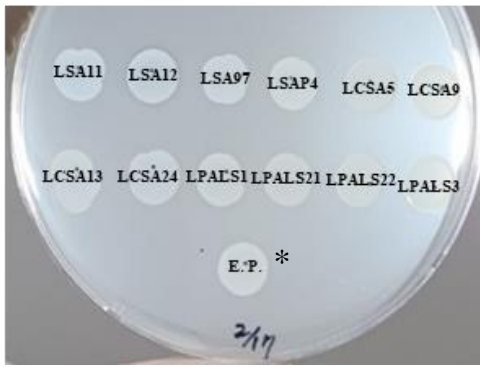

*E.P.: empty plasmid (pET28a ::no endolysin + pBAD33::SPN1S_lysRz/ BL21(DE3) star)

Figure S2. Validation of the screening system with 12 positive controls.

(A) Lysis zones of 12 natural staphylococcal endolysins after induction of IPTG (under $30{ }^{\circ} \mathrm{C}$ for $6 \mathrm{~h}$ ) and arabinose.

(B) Lysis zones of 12 natural staphylococcal endolysins after induction of IPTG (under $18{ }^{\circ} \mathrm{C}$ for $20 \mathrm{~h}$ ) and arabinose.

(C) Lysis zones of 12 natural staphylococcal endolysins after arabinose induction without IPTG induction (SPN1S_lysRz expression without endolysin expression). 
A

S. aureus $\mathrm{RN} 4220$

(20 mM Tris-HCl, pH 8.0)

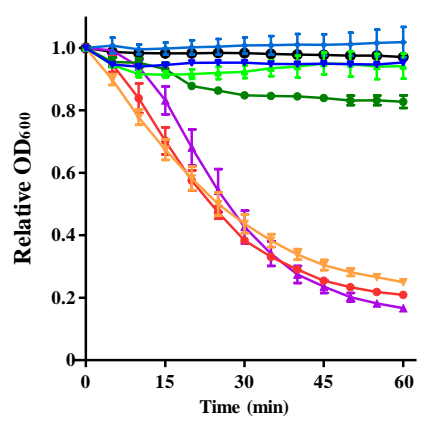

B

S. aureus ATCC33593 (50 mM Tris-HCl, pH 7.5)

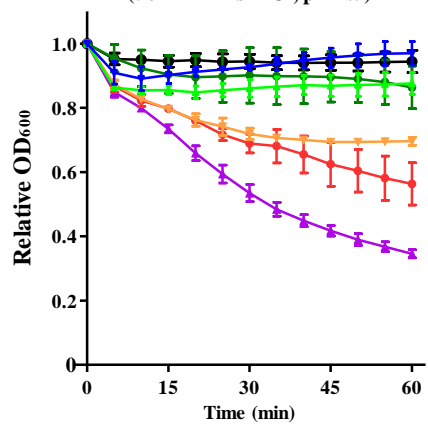

C

S. aureus ATCC33586

(50 mM Tris-HCl, pH 7.5)

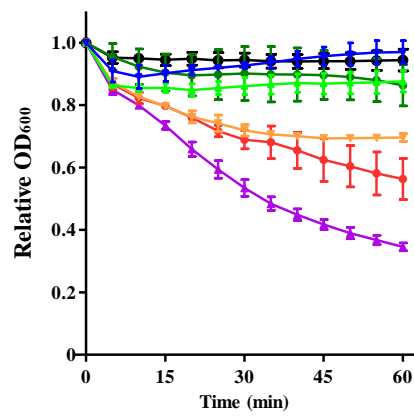

$\rightarrow$ Control

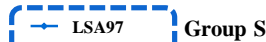
$\rightarrow$ LPALS22 (Small lysis zone)

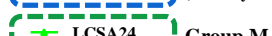
$\rightarrow$ LSAP4 (Medium lysis zone)

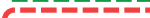
$\because$ LCSA13 Group L $\rightarrow$ LSA12 (Late L

Figure S3. Lytic activity of 6 staphylcococcal endolysins and ClyC.

Turbidity reduction assay of 6 natural staphylococcal endolysins (LysSA97, LysPALS22, LysCSA24, LysSAP4, LysCSA13, and LysSA12) and ClyC against various staphylococcal strains was conducted. For the turbidity reduction assay, $500 \mathrm{nM}$ of endolysins were treated against (A) S. aureus RN4220 in reaction buffer (20 mM Tris-HCl, $\mathrm{pH} 8.0$ ), (B) S. aureus ATCC33593 in reaction buffer (50 mM Tris-HCl, pH 7.5), and (C) S. aureus ATCC33586 in reaction buffer (50 mM Tris-HCl, $\mathrm{pH} 7.5)$. 


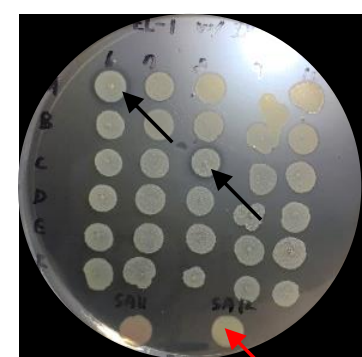

Library 1 (1A1-1F5)

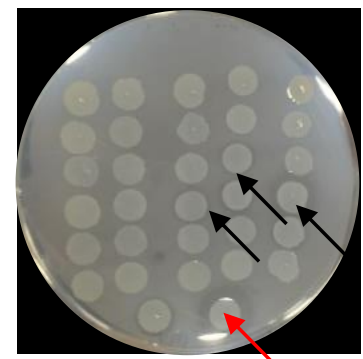

Library 6 (6A1-6F5)

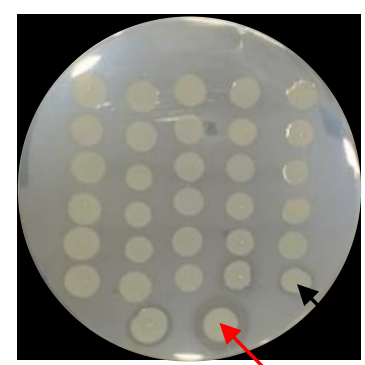

Library 8 (8A1-8F5)

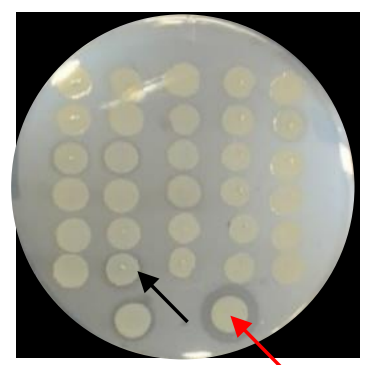

Library 10 (10A1-10F5)

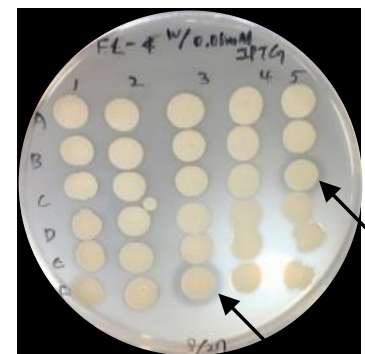

Library 4 (4A1-4F5)

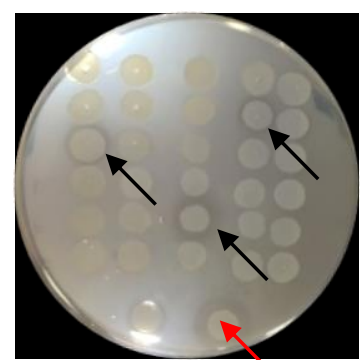

Library 6 (6A6-6F10)

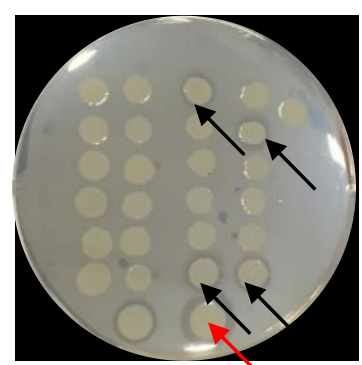

Library 8 (8A6-8F10)

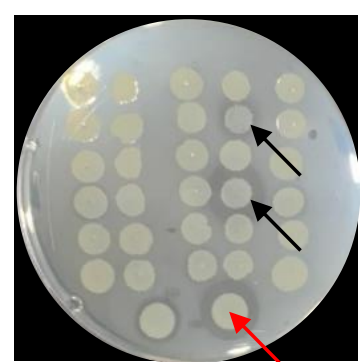

Library 10 (10A6-10F10)

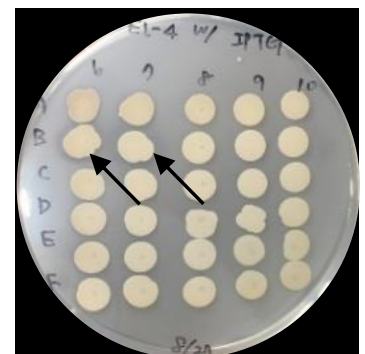

Library 4 (4A6-4F10)

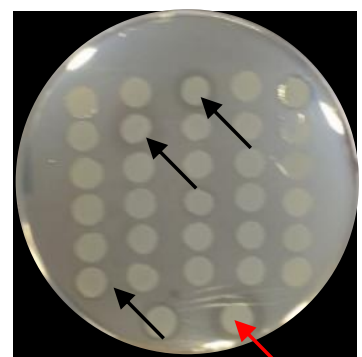

Library 7 (7A1-7F5)

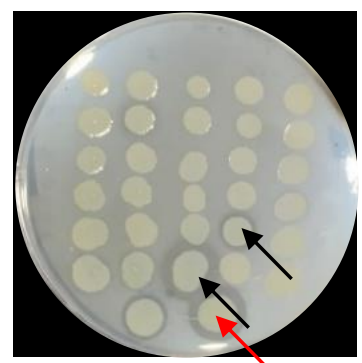

Library 9 (9A1-9F5)

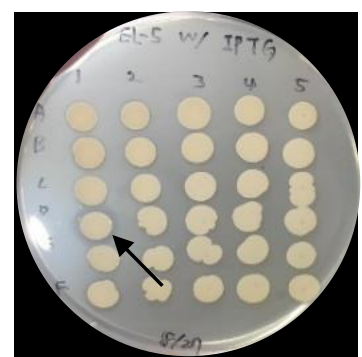

Library 5 (5A1-5F5)

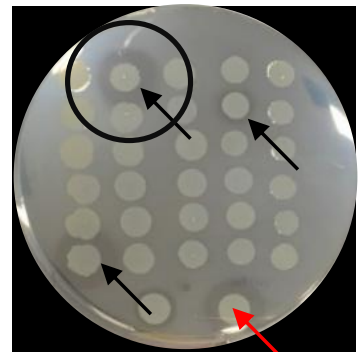

Library 7 (7A6-7F10)

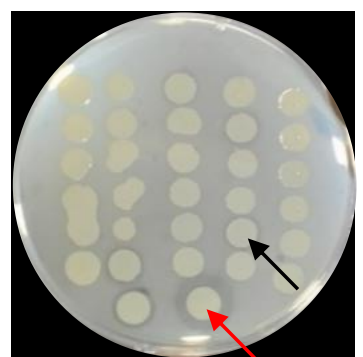

Library 9 (9A6-9F10)

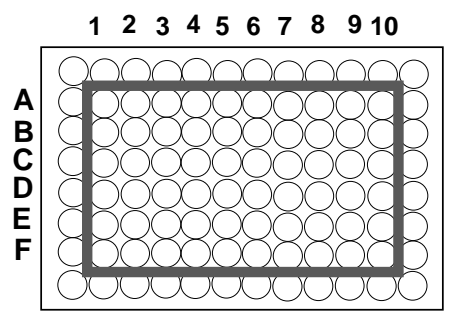

Figure S4. Lysis zone-based screening results.

For first screening, lysis zone-based screening was performed in a qualitative manner and 30 candidate chimeric endolysins were selected as indicated as black arrows in above pictures. Positive control (LysSA12) was marked with red arrows and ClyC is marked as black circle in library 7 (7A7). 
A

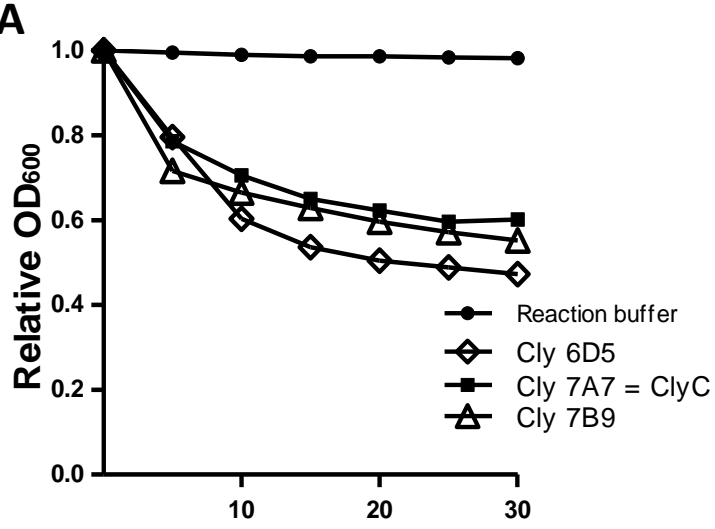

Time (min)

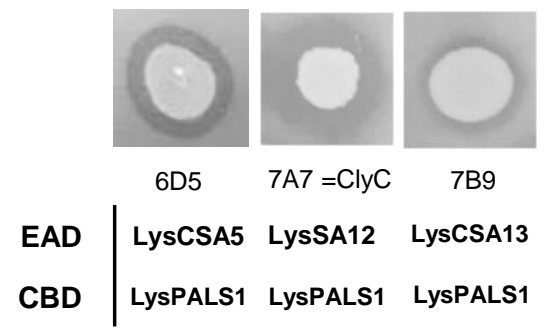

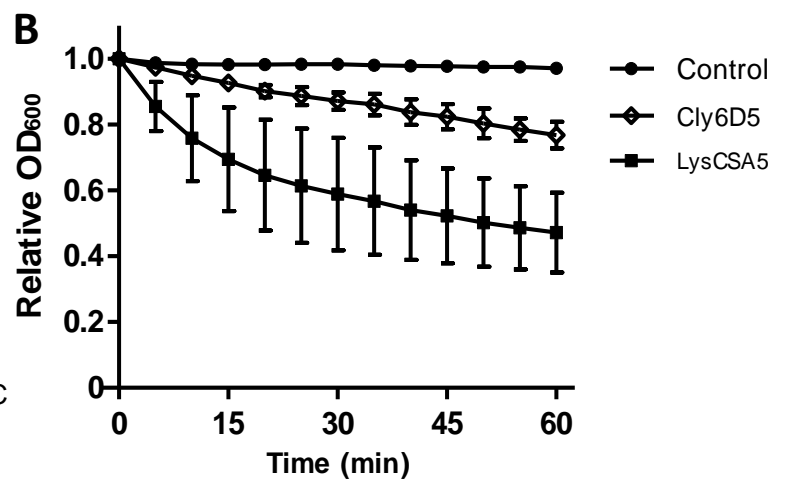

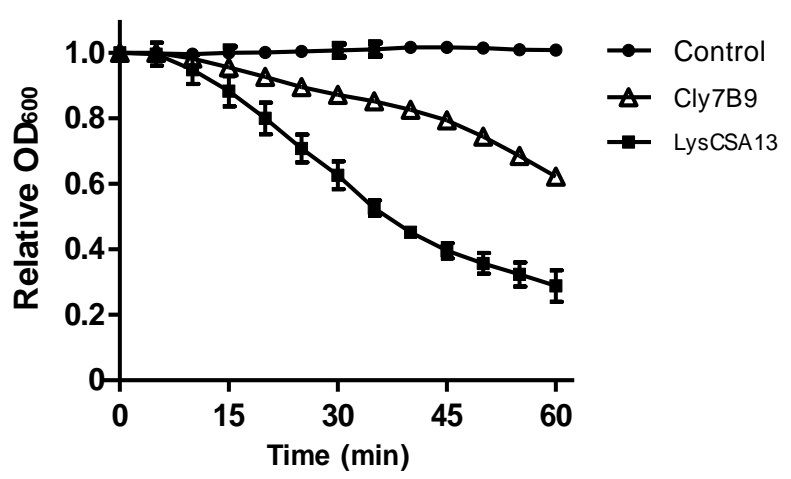

Figure S5. Quantitative analysis of lytic activity for candidate chimeolysins.

(A) Three chimeric endolysins which displayed high lytic activity were selected and the relative lytic activity and lysis zone against $S$. aureus RN4220 were compared. (B) The relative OD ${ }_{600}$ reduction of two chimeric endolysins (6D5 and 7B9) and its parental endolysins (LysCSA5 and LysCSA13, respectively) against $S$. aureus RN4220 was compared. Turbidity reduction assay was conducted with $100 \mathrm{nM}$ of endolysin after purification with affinity chromatography. 
A

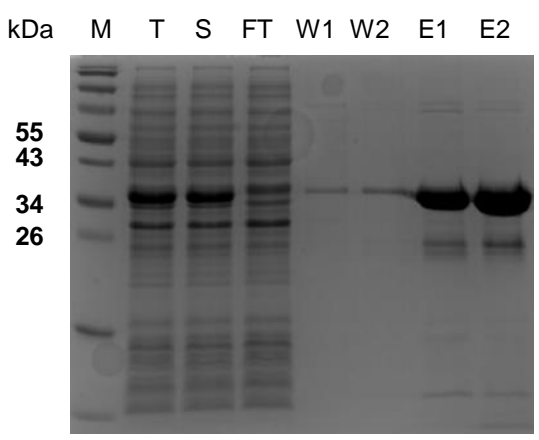

B

$\begin{array}{llllllllll}k D a & M & T & S & F T & \text { W1 } & \text { W2 } & \text { E1 } & \text { E2 }\end{array}$

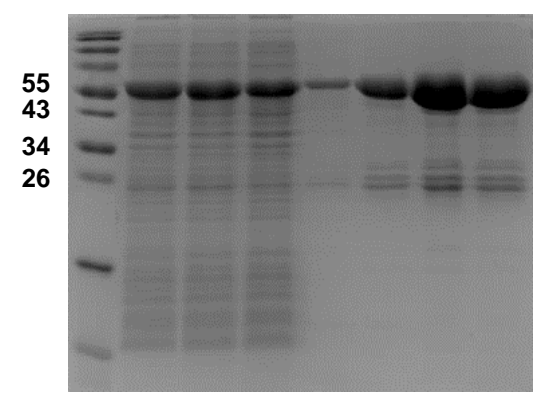

C

$\begin{array}{llllllllll}\mathrm{kDa} & \mathrm{M} & \mathrm{T} & \mathrm{S} & \mathrm{FT} & \mathrm{W} 1 & \mathrm{~W} 2 & \mathrm{E} 1 & \mathrm{E} 2\end{array}$

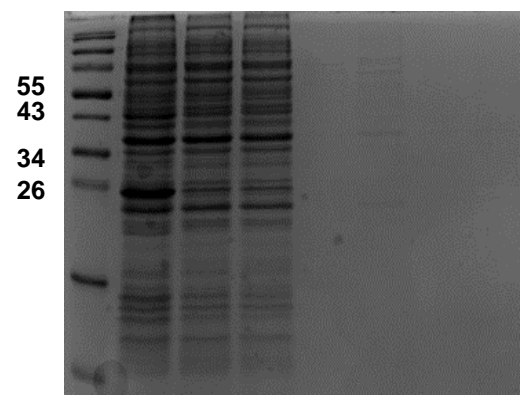

Figure S6. SDS-PAGE analysis of ClyC and its parental endolysins.

(A) Induced expression of ClyC (34.7 kDa) on $15 \%$ SDS-PAGE gel. (B) Induced expression of LysSA12 (57.5 kDa) on $15 \%$ SDS-PAGE gel. (C) Induced expression of LysPALS1 (26.6 kDa, induction under $30^{\circ} \mathrm{C}$ ) on $15 \%$ SDS-PAGE gel. $10 \mu \mathrm{g}$ of each Ni-NTA purified protein was loaded per lane. Lanes; M: marker, T: total protein, S: soluble protein, FT: flow through after Nickel-his tag binding, W1: washing with $10 \mathrm{mM}$ imidazole, W2: $20 \mathrm{mM}$ imidazole, E1: elution with $150 \mathrm{mM}$ imidazole, E2: $200 \mathrm{mM}$ imidazole. 
A

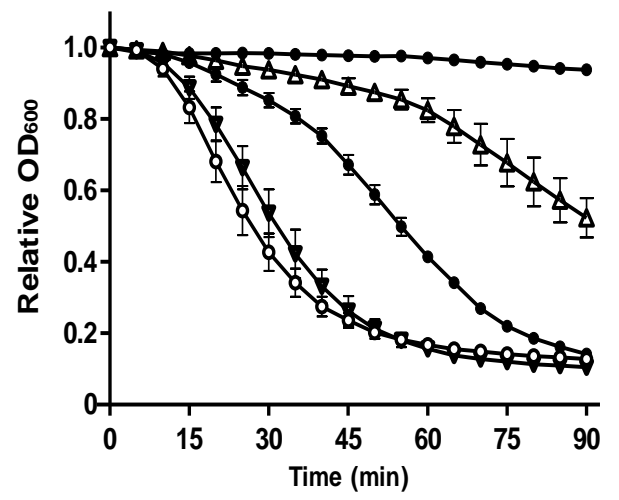

B

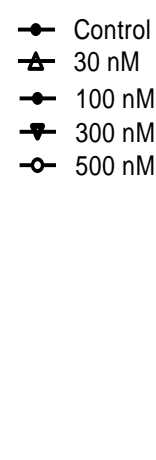

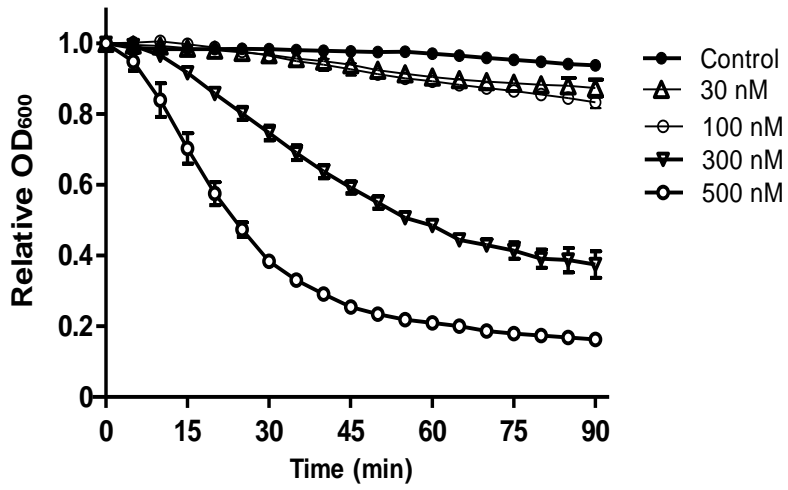

Figure S7. Dose-dependent turbidity reduction ability of ClyC and LysSA12. Turbidity reduction assay of (A) ClyC and (B) LysSA12 against S. aureus RN4220 with diverse endolysin concentrations $(0,30,100,300,500 \mathrm{nM})$ was presented. S. aureus RN4220 was washed with reaction buffer (20 mM Tris- $\mathrm{Cl}, \mathrm{pH} 8.0)$ and the change of $\mathrm{OD}_{600}$ was monitored by the microplate reader at $30{ }^{\circ} \mathrm{C}$ for $90 \mathrm{~min}$. 


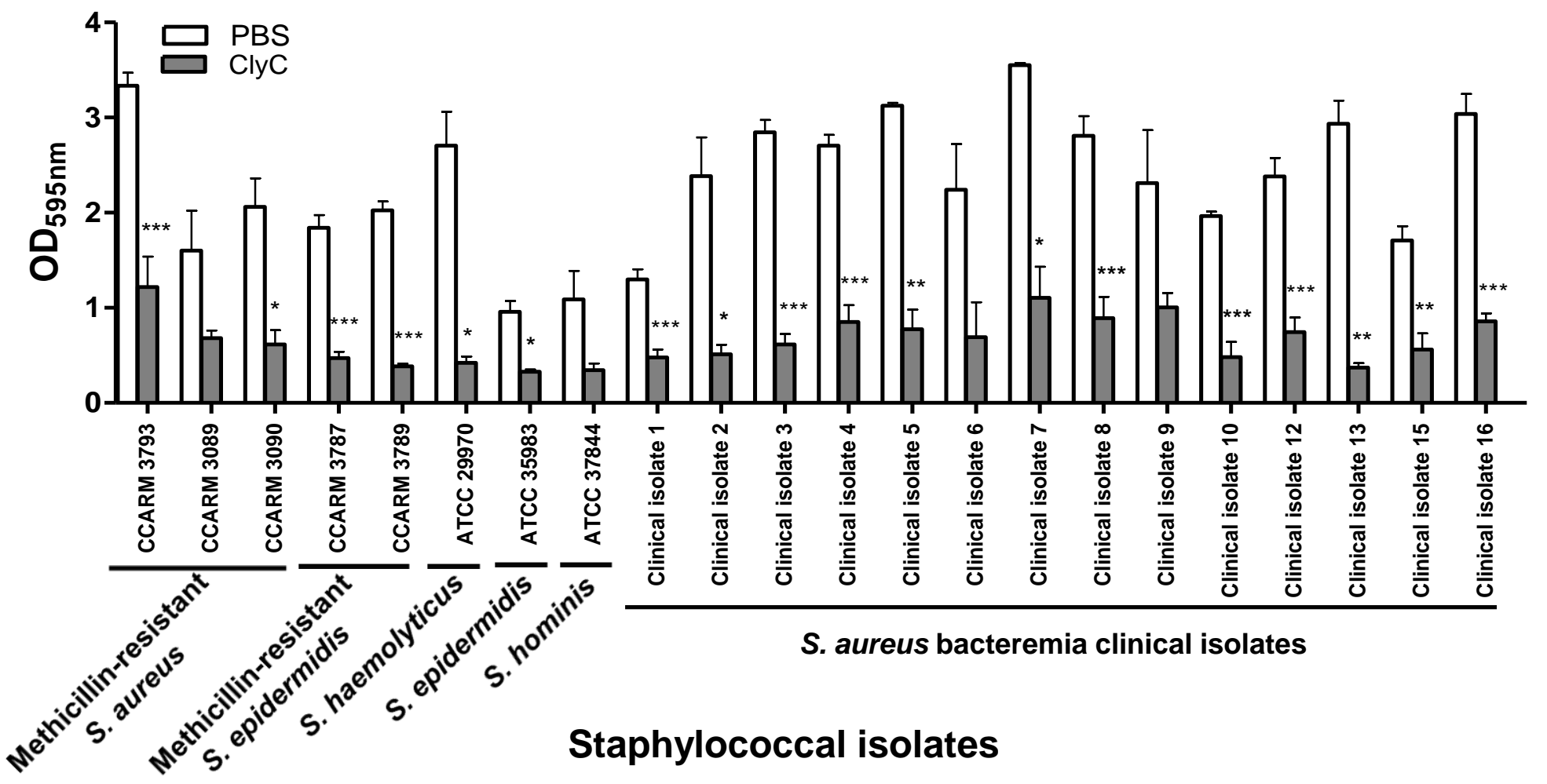

Figure S8. Biofilm disrupting ability of ClyC against diverse staphylococcal isolates.

After treatment of ClyC, residual biofilms of 22 staphylococcal strains, including MRSA, MRSE, and other S. aureus clinical isolates, were measured. Total $1 \mu \mathrm{M}$ of ClyC was treated to each strains for $2 \mathrm{~h}$. Biofilm reduction assay was conducted over three times and data were analyzed based on the negative control by t-test. $* \mathrm{p}<0.05, * * \mathrm{p}<0.01, * * * \mathrm{p}<0.005$. 


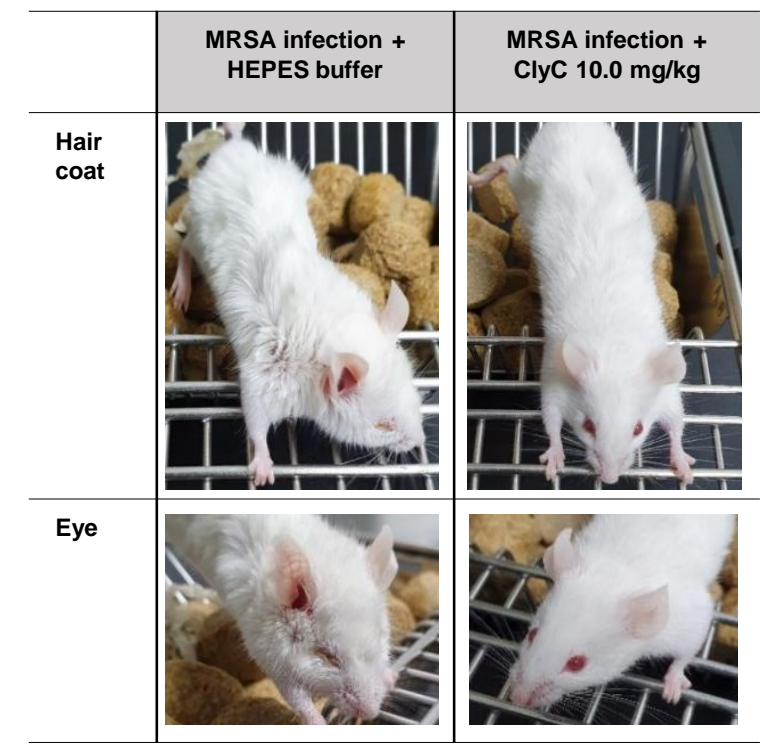

Figure S9. Effect of ClyC on the severity of MRSA bacteremia in mice after $24 \mathrm{~h}$ injection. After $1 \mathrm{~h}$ MRSA injection, health states of mice treated with a reaction buffer $(20 \mathrm{mM}$ HEPES buffer, $\mathrm{pH} 7.4)$ and $\mathrm{ClyC}(10.0 \mathrm{mg} / \mathrm{kg})$ were observed and compared. 
Amino acid sequences

from LysSA12

Amino acid sequences from LysPALS1
MQAKLTKKEFIEWLKTSEGKQYNADGWYGFQCFDYANAGWQVLFGYNLK

GVGAKDIPSANDFNGLATVYQNTPDFLAQPGDMVVFGSNYGAGYGHVAW

VIEATLDYIIVYEQNWLGGGWTDGVQQPGSGWEKVTRRQHAYDFPMWFIR

PNFKSETAPRSVQSPTQASKKETVDSASTPATRPVTGSWKKNQYGTWYKPE

SATFVNGNQPIVTRIGSPFLNAPVGGNLPAGATIVYDEVCIQAGHIWIGYNAY

NGNRVYCPVRTCQGVPPSHVPGVAWGTFK

CHAP domain
SH3 domain

Figure S10. Amino acid sequences of ClyC investigated in this study. 
Table S1. Plasmids used in this paper

\begin{tabular}{|c|c|}
\hline Plasmid & Description \\
\hline pBAD33 & $\mathrm{Amp}^{\mathrm{R}}$, araC, arabinose promoter, $\mathrm{pBR} 322_{\text {ori }}$ \\
\hline pBAD33::SPN1S_lysRz & $\mathrm{Amp}^{\mathrm{R}}$, araC, arabinose promoter, $\mathrm{pBR} 322_{\text {ori }} \cdot \mathrm{SPN} 1 \mathrm{~S}-\mathrm{lysRz}$ gene \\
\hline pET28a & $\mathrm{Kan}^{\mathrm{R}}$, T7 lac promoter, 6 His-tagged expression vector \\
\hline pET28a::6D5 & $\mathrm{Kan}^{\mathrm{R}}$, T7 lac promoter, 6 His-tagged expression vector, LysCSA5 CHAP-LysPALS1 CBD gene \\
\hline pET28a::ClyC & $\mathrm{Kan}^{\mathrm{R}}$, T7 lac promoter, 6 His-tagged expression vector, LysSA12 CHAP-LysPALS1 CBD gene \\
\hline pET28a::7B9 & $\mathrm{Kan}^{\mathrm{R}}$, T7 lac promoter, 6 His-tagged expression vector, LysCSA13 CHAP-LysPALS1 CBD gene \\
\hline pET28a::LysSA11 & $\mathrm{Kan}^{\mathrm{R}}$, T7 lac promoter, 6 His-tagged expression vector, LysSA11 gene \\
\hline pET28a::LysSA12 & $\mathrm{Kan}^{\mathrm{R}}$, T7 lac promoter, 6 His-tagged expression vector, LysSA12 gene \\
\hline pET28a::LysPALS1-1 & $\mathrm{Kan}^{\mathrm{R}}$, T7 lac promoter, 6 His-tagged expression vector, N-terminal His tag, LysPALS1 gene \\
\hline pET28a::LysPALS1-2 & $\mathrm{Kan}^{\mathrm{R}}$, T7 lac promoter, 6 His-tagged expression vector, C-terminal His-tag, LysPALS1 gene \\
\hline
\end{tabular}


Table S2. Staphylococcal endolysins used in this study and domain identification through BLAT, HMMER, and NPSA-Prabi tools.

\begin{tabular}{|c|c|c|c|c|c|c|c|}
\hline No. & Endolysin & $\begin{array}{c}\text { Mw } \\
\text { (kDa) }\end{array}$ & pI & \multicolumn{2}{|c|}{ EAD } & CBD & Accession No. \\
\hline 1 & LysSA11 & 28.8 & 9.27 & \multicolumn{2}{|r|}{ CHAP } & N.D. ${ }^{a)}$ & GenBank.JX194239.1 \\
\hline 2 & LysSA97 & 54 & 8.87 & \multicolumn{2}{|c|}{ CHAP, MurNAc-LAA(ami3) } & N.D. & GenBank. KJ716334 \\
\hline 3 & LysSA12 & 54 & 8.7 & CHAP & PGRP(ami2) & SH3_5 & GenBank. KC677663 \\
\hline 4 & LysSAP4 & 54 & 9.27 & CHAP & MurNAc-LAA(ami3) & $\mathrm{SH} 3 \mathrm{~b}$ & N.A. b) \\
\hline 5 & LysCSA13 & 28 & 6.81 & \multicolumn{2}{|r|}{ CHAP } & SH3_5 & GenBank. MH107118 \\
\hline 6 & LysCSA9 & 57 & 9.27 & CHAP & MurNAc-LAA(ami3) & $\mathrm{SH} 3 \mathrm{~b}$ & N.A. \\
\hline 7 & LysPALS21 & 29 & 9.97 & \multicolumn{2}{|r|}{ CHAP } & $\mathrm{SH} 3$ & N.A. \\
\hline 8 & LysPALS22 & 35.0 & 9.40 & \multicolumn{2}{|c|}{ PGRP(ami2) } & $\mathrm{SH} 3$ & N.A. \\
\hline 9 & LysPALS3 & 29.0 & 9.25 & \multicolumn{2}{|r|}{ CHAP } & N.D. & N.A. \\
\hline 10 & LysCSA5 & 54.8 & 9.53 & CHAP & PGRP(ami2) & $\mathrm{SH} 3$ & GenBank. JQ686190.1 \\
\hline 11 & LysCSA24 & 28.6 & 6.41 & \multicolumn{2}{|r|}{ CHAP } & $\mathrm{SH} 3$ & N.A. \\
\hline 12 & LysPALS1 & 23.0 & 9.92 & \multicolumn{2}{|r|}{ N.D. } & $\mathrm{SH} 3$ & N.A. \\
\hline
\end{tabular}

a) Not Detected in BLAST (Basic Local Alignment Search Tool) search.

b) Accession number is not available. 
Table S3. Primers used in this study for the construction of recombinant endolysin library.

\begin{tabular}{|c|c|c|c|}
\hline Primer & Sequence $\left(5^{\prime}-3^{\prime}\right)$ & $\operatorname{mer}(\mathbf{s})$ & $T_{m}$ value \\
\hline LSA11CHAP_BamH1_F & 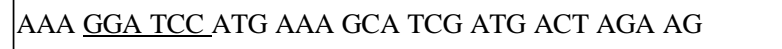 & 32 & 59.2 \\
\hline LSA11CHAP_Sal1_R & TTT GTC GAC TCC ACC TAA CCA ATT ATT TTC AAT TA & 35 & 58.4 \\
\hline LSA11CBD_Sal1_F & AAA GTC GAC GGT GGC TGG GAG AAA GCT & 27 & 58.4 \\
\hline LSA11CBD_HindIII_R & 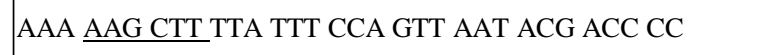 & 32 & 59.2 \\
\hline LSA12CHAP_BamH1_F & AAA GGA TCC ATG CAA GCA AAA CTA ACT AAA AAA GA & 35 & 58.4 \\
\hline LSA12CHAP_Sal1_R & TTT GTC GAC CGT TTC CTT TTT AGA TGC TTG C & 31 & 58.4 \\
\hline LSA12Ami_BamH1_F & AAA GGA TCC ATG GTA CAA CAA CCT GGC TCT & 30 & 59.5 \\
\hline LSA12Ami_Sal1_R & TTT GTC GAC AGC GCT TGA CTC ATT AGA GAC & 30 & 59.5 \\
\hline LSA12CBD_Sal1_F & AAA GTC GAC CTA TTG CCA GAA GAC AAG CG & 29 & 58.4 \\
\hline LSA12CBD_HindIII_R & TTT AAG CTT CTA ACT GAT TTC TCC CCA TAA G & 31 & 58.4 \\
\hline LSA97CHAP_BamH1_F & AAA GGA TCC ATG CCG TCG GTT AGG ACA TA & 29 & 58.4 \\
\hline LSA97CHAP_Sal1_R & TTT GTC GAC TGC GCT TCT TAG CTT ATT AGC & 30 & 57.5 \\
\hline LSA97Ami_BamH1_F & AAA GGA TCC ATG ACT ACA AGA CGC ACA CAC & 30 & 59.5 \\
\hline LSA97Ami_Sal1_R & TTT GTC GAC GCT TGG CTT ACT ACT TGG C & 28 & 57.5 \\
\hline LSA97CBD_Sal1_F & AAA GTC GAC AGT AAG CCA AGC GCT GAC A & 28 & 57.5 \\
\hline LSA97CBD_HindIII_R & TTT AAG CTT CGT GCC CCA ATA TTT TTC ATT TTT & 33 & 58.4 \\
\hline LSAP4CHAP_BamH1_F & AAA $\underline{\text { GGA TCC }}$ ATG ACA AAA AAC CAA GCA GAA AAA T & 34 & 57.6 \\
\hline LSAP4CHAP_Sal1_R & TTT GTC GAC ACT TAC TTT ATC TGG GAA ATT TAA TC & 35 & 58.4 \\
\hline LSAP4Ami_BamH1_F & AAA GGA TCC ATG GCG CAA CCT GGT TGG & 27 & 58.4 \\
\hline LSAP4Ami_Sal1_R & TTT GTC GAC TGC TGG CAC TGG TGG ATT TT & 29 & 58.4 \\
\hline LSAP4CBD_Sal1_F & AAA GTC GAC GGT TTG GTA GCT GGT AAT GTT A & 31 & 58.4 \\
\hline LSAP4CBD_HindIII_R & 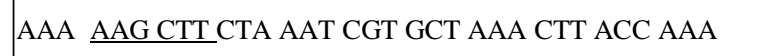 & 33 & 58.3 \\
\hline LCSA5CHAP_BamH1_F & AAA GGA TCC ATG GCT AAG ACT CAA GCA GAA A & 31 & 58.4 \\
\hline LCSA5CHAP_Sal1_R & TTT GTC GAC TGC ACT TTT CTT AGC TGT TTC TTT & 33 & 58.3 \\
\hline LCSA5Ami_BamH1_F & AAA $\underline{\text { GGA TCC }}$ ATG AAT AAA AAA CCT ACA AAA CGT G & 34 & 57.6 \\
\hline LCSA5Ami_Sal1_R & TTT GTC GAC TGC GCT ACT TGT TTT ACC GTC & 30 & 59.5 \\
\hline LCSA5CBD_Sal1_F & AAA GTC GAC ACA CAA GGA AGA CCA TCT CAA & 30 & 57.5 \\
\hline LCSA5CBD_HindIII_R & TTT AAG CTT CTA TTT GAA TAC TCC CCA AGC A & 31 & 58.4 \\
\hline
\end{tabular}




\begin{tabular}{|c|c|c|c|}
\hline Primers & Sequence $\left(5^{\prime}-3^{\prime}\right)$ & $\operatorname{mer}(\mathbf{s})$ & $\mathbf{T}_{\mathbf{m}}$ value \\
\hline LCSA9CHAP_BamH1_F & AAA GGA TCC ATG TTA ATG ACA AAA AAT CAA GCA GA & 35 & 58.4 \\
\hline LCSA9CHAP_Sal1_R & TTT GTC GAC GCT TAA GTT GTT AGG GAA GTT TAA & 33 & 58.3 \\
\hline LCSA9Ami_BamH1_F & AAA GGA TCC ATG GTT GCG CAA CCT GGT T & 28 & 57.5 \\
\hline LCSA9Ami_Sal1_R & TTT GTC GAC TGC TGG CAC TGG TGG ATT TT & 29 & 58.4 \\
\hline LCSA9CBD_Sal1_F & AAA GTC GAC GCT AAA AAC AAA AAA AAT CCA CCA G & 34 & 59.2 \\
\hline LCSA9CBD_HindIII_R & 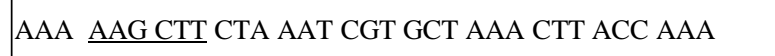 & 33 & 58.3 \\
\hline LCSA13CHAP_BamH1_F & AAA GGA TCC ATG AAA TCA CAA AAA CAA GCA AAA C & 34 & 57.6 \\
\hline LCSA13CHAP_Sal1_R & TTT GTC GAC TAC TTG ACT ATT ACT GTC TGA AAA T & 34 & 57.6 \\
\hline LCSA13CBD_Sal1_F & AAA GTC GAC GGA TAT GAT GGT TGG GAA AAA G & 31 & 58.4 \\
\hline LCSA13CBD_HindIII_R & TTT AAG CTT TTA TGA GAA CAC CCC CCA AG & 29 & 58.4 \\
\hline LCSA24CHAP_BamH1_F & AAA GGA TCC ATG AAA TCA CAA CAA CAA GCG AAA & 33 & 58.3 \\
\hline LCSA24CHAP_Sal1_R & TTT GTC GAC TAC TTT ACT TTC ACT GTT TGA GAA T & 34 & 57.6 \\
\hline LCSA24CBD_Sal1_F & AAA GTC GAC GGA TAT GAT GGT TGG GAA AAA G & 31 & 58.4 \\
\hline LCSA24CBD_HindIII_R & TTT AAG CTT TTA TGA GAA CAC CCC CCA AG & 29 & 58.4 \\
\hline LPALS1CHAP_BamH1_F & AAA GGA TCC ATG AGT GCT AGT GAT GCT CAA T & 31 & 58.4 \\
\hline LPALS1CHAP_Sal1_R & TTT GTC GAC ACC TGT AAC TGG TCT AGT TGC & 30 & 59.5 \\
\hline LPALS1CBD_Sal1_F & AAA GTC GAC AGT GCA AGT ACA CCG GCA A & 28 & 57.5 \\
\hline LPALS1CBD_HindIII_R & TTT AAG CTT CTA CTT GAA TGT TCC CCA GG & 29 & 58.4 \\
\hline LPALS21CHAP_BamH1_F & TTT GGA TCC ATG AAA ACT AAA ACT CAA GCT TTG AA & 35 & 58.4 \\
\hline LPALS21CHAP_Sal1_R & TTT GTC GAC TGA ATT ACG TTT CCA TTT AGC AGA & 33 & 58.3 \\
\hline LPALS21CBD_Sal1_F & AAA GTC GAC AGC AAT GGT TCA CCT GCA G & 28 & 57.5 \\
\hline LPALS21CBD_HindIII_R & 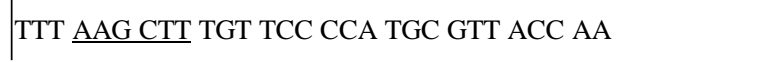 & 29 & 58.4 \\
\hline LPALS22CHAP_BamH1_F & AAA GGA TCC ATG GCT AAA AAA CAT ATC GGT ACA & 33 & 58.3 \\
\hline LPALS22CHAP_Sal1_R & TTT GTC GAC ACT TGT TTT CTT AGG TTT AGC AG & 32 & 57.6 \\
\hline LPALS22CBD_Sal1_F & AAA GTC GAC GCA TGC GGT ATG TCT CGT A & 28 & 57.6 \\
\hline LPALS22CBD_HindIII_R & TTT AAG CTT TGT TCC CCA TGC TGG ACC & 27 & 58.4 \\
\hline LPALS3CHAP_BamH1_F & AAA GGA TCC ATG AAA ACA TAC AGT GAA GCA AGA & 33 & 58.3 \\
\hline LPALS3CHAP_Sal1_R & TTT GTC GAC GCC CTC ACT TTT ATA CTT AGG T & 31 & 58.4 \\
\hline LPALS3CBD_Sal1_F & AAA GTC GAC AAC GCA AAT ACG CCT GCA AAG & 30 & 59.5 \\
\hline LPALS3CBD_HindIII_R & 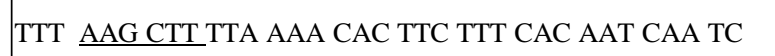 & 35 & 58.4 \\
\hline
\end{tabular}


Table S4. Domain identification of 15 chimeric endolysin candidates.

After sequencing 30 chimeric endolysins which showed large lysis zones in a two vector-based screening step, 15 chimeric endolysins were identified as having different domain structures. Those 15 chimeric endolysins were further confirmed its antibacterial activity under turbidity reduction assay with soluble forms.

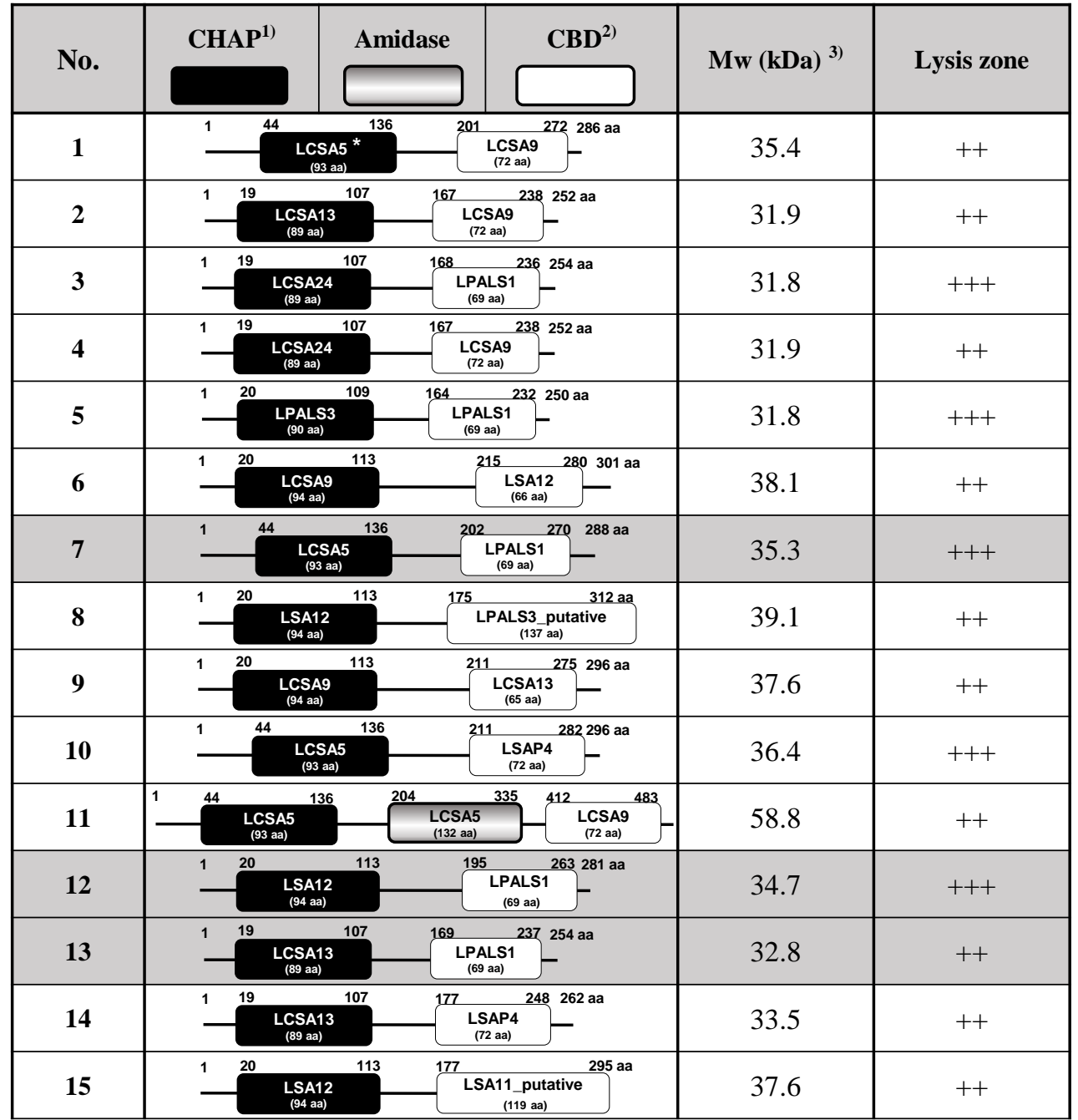

1)CHAP: Cysteine, Histidine-dependent Amidohydrolase/ Peptidase

${ }^{2)} \mathrm{CBD}$ : Cell wall Binding Domain

${ }^{3)} \mathrm{Mw}$ : Molecular weight

* LCSA5: LysCSA5, indicating endolysin from staphylococcal phage CSA5 